\title{
Enhanced Ex Vivo Expansion of Human Adipose Tissue-Derived Mesenchymal Stromal Cells by Fibroblast Growth Factor-2 and Dexamethasone
}

\author{
Sun-Young Lee, M.S., ${ }^{1, \star}$ Jiwon Lim, M.S., ${ }^{2, *}$ Gilson Khang, Ph.D., ${ }^{3}$ Youngsook Son, Ph.D., ${ }^{4}$ \\ Phil-Hoon Choung, D.D.S., Ph.D., ${ }^{5}$ Shin-Sung Kang, Ph.D., ${ }^{6}$ So Young Chun, Ph.D., ${ }^{2}$ \\ Hong-In Shin, D.D.S., Ph.D., ${ }^{2}$ Shin-Yoon Kim, M.D., Ph.D., ${ }^{1,7}$ and Eui Kyun Park, Ph.D. ${ }^{1,2}$
}

In the present study, we investigated the ex vivo expansion of human adipose tissue-derived mesenchymal stromal cells (ATSCs) to identify factors that promoted efficient expansion while preserving stem cell potential. We examined several growth factors and steroids, and found that the combination of a low concentration of fibroblast growth factor-2 (FGF-2) $(1 \mathrm{ng} / \mathrm{mL}$ ) and dexamethasone (DEX) or betamethasone (BET) enhanced the proliferation of ATSCs by approximately $30-60 \%$ as compared to control. Enhanced proliferation under these conditions was confirmed using ATSCs isolated from three independent donors. ATSCs that were expanded in the presence of FGF-2 and DEX for 5 days were capable of differentiating into either osteoblastic or adipogenic cells, and the cells were positive for the mesenchymal stem cell markers such as CD29, CD44, CD90, CD105, and CD146, suggesting that the stem cell potential of the ATSCs was preserved. Analysis of signaling pathway revealed that tyrosine phosphorylation of Src kinase was dramatically increased in response to FGF-2 and DEX, suggesting the involvement of Src-dependent pathways in the stimulatory mechanism of proliferation of ATSCs by FGF-2 and DEX. Moreover, Src family kinase inhibitors (SU6656 and Src kinase inhibitor I) substantially reduced the FGF-2 and DEX-induced proliferation of ATSCs. SU6656 also inhibited the osteogenic and adipogenic differentiation of ATSCs. The results of the current study demonstrate that FGF-2 in combination with DEX stimulates the proliferation and osteoblastic and adipogenic differentiation of ATSCs through a Src-dependent mechanism, and that FGF-2 and DEX promote the efficient ex vivo expansion of ATSCs.

\section{Introduction}

$\mathbf{T}$ ISSUE ENGINEERING USING mesenchymal stem cells (MSCs) is a promising technology for the treatment or regeneration of tissue defects. MSCs have been identified in a variety of tissues, including adipose tissue. ${ }^{1-3}$ Adipose tissue is a valuable source of MSC because it is abundant and can be obtained with little surgical difficulty. Under the appropriate conditions, MSCs derived from adipose tissue are capable of differentiating into adipogenic, chondrogenic, myogenic, and osteogenic cells. ${ }^{4}$ The cell surface expression of MSC markers on adipose tissue-derived mesenchymal stromal cells (ATSCs) is similar to that of bone marrow- derived mesenchymal stem cells (BMSCs). ${ }^{5}$ Moreover, ATSCs are similar to BMSCs in their potential to differentiate into osteoblasts in vitro and to synthesize bone in vivo. ${ }^{6}$ ATSCs, in combination with recombinant bone morphogenetic proteins, have been shown to induce bone formation in vivo and promote healing of a critically sized femoral defect in an athymic rat. ${ }^{7}$ These results indicate that ATSCs are a viable alternative source of stem cells for bone regeneration. However, to obtain reasonable numbers of ATSCs for tissue engineering applications-in particular, the regeneration of large lesions-ex vivo expansion of ATSCs will be required.

Several growth factors have been implicated in the regulation of MSC proliferation. In particular, fibroblast growth

\footnotetext{
${ }^{1}$ Skeletal Diseases Genome Research Center, Kyungpook National University Hospital, Daegu, Republic of Korea.

${ }^{2}$ Department of Pathology and Regenerative Medicine, IHBR, School of Dentistry, Kyungpook National University, Daegu, Republic of Korea.

${ }^{3}$ Department of Polymer-Nano Science and Technology, Chonbuk National University, Jeonju, Republic of Korea.

${ }^{4}$ School of Life Science, Kyung Hee University, Yong In, Republic of Korea.

${ }^{5}$ Department of Craniofacial Surgery, School of Dentistry, Seoul National University, Seoul, Republic of Korea.

${ }^{6}$ Department of Biology, College of Natural Sciences; ${ }^{7}$ Department of Orthopaedic Surgery, School of Medicine; Kyungpook National University, Daegu, Republic of Korea.

*Both authors contributed equally to this work.
} 
factors (FGFs) regulate multiple biological processes, including bone and cartilage formation. ${ }^{8}$ The effect of FGF-2 on MSC proliferation varies, and depends largely on the stage of differentiation of the MSCs. ${ }^{9-11}$ Similarly, variable effects of FGF2 on the osteoblastic differentiation of BMSCs have also been reported. FGF-2 significantly increases the proliferation of ATSCs and enhances chondrogenesis in a three-dimensional micromass culture. ${ }^{12}$ However, FGF-2 also reportedly inhibits osteogenesis in mouse ATSCs and sustains their proliferative and osteogenic potential. ${ }^{13}$ Recently, FGF-2 has been shown to maintain the self-renewing state human ATSCs. ${ }^{14}$

Steroids can regulate multiple biological processes, including bone formation. Dexamethasone (DEX), a synthetic corticosteroid, is a potent modulator of the osteogenic differentiation of MSCs. DEX supports osteogenic lineage differentiation ${ }^{15-17}$ by binding to specific regulatory proteins and activating the transcription of osteoblast-specific genes. DEX increases alkaline phosphatase (ALP) activity, which is required for matrix mineralization. ${ }^{16}$ The combination of FGF and DEX induces more profound effects on the osteoblastic differentiation of MSCs derived from bone marrow. For example, FGF-2 in combination with DEX increases the colony size and DNA content of human BMSCs as compared to FGF-2 alone. However, the underlying molecular mechanism of action of the two factors is unknown. ${ }^{18-20}$ BMSCs that are expanded in the presence of FGF-2 and DEX stimulate bone formation in nude mice. ${ }^{20}$ In some cases, such as with rat marrow stromal cells, treatment with FGF-2 and DEX decreases DNA content as compared to FGF-2 alone. ${ }^{21}$

In the present study, we developed an efficient method for the ex vivo expansion of ATSCs using FGF-2 in combination with steroids, and showed that ATSCs that are expanded in the presence of FGF-2 and DEX maintain their multi-lineage potential. We also investigated the stimulatory mechanism of proliferation of ATSCs by FGF-2 and DEX.

\section{Materials and Methods}

\section{Isolation and culture of ATSCs}

Fat tissue was obtained from patients undergoing total hip replacement surgery. Informed consent was obtained from the patients before surgery. Fat tissue was minced with a scalpel and washed with phosphate buffered saline ( $\mathrm{pH} 7.4)$ to remove contaminating blood, and then digested with collagenase type I $2 \mathrm{mg} / \mathrm{mL}$ (Warthington Biochemical, Lakewood, NJ) for $45 \mathrm{~min}$ at $37^{\circ} \mathrm{C}$ with constant agitation. Enzyme activity was neutralized by the addition of $\alpha$-modified Eagle's medium ( $\alpha$-MEM) (Gibco BRL, Gaithersburg, MD) containing $10 \%$ fetal bovine serum (FBS) (Biowhittaker, Walkersville, MD). Cells were obtained by centrifugation at $1200 \mathrm{~g}$ for $10 \mathrm{~min}$. Cell pellets were resuspended in $\alpha$-MEM and filtered through a $250-\mu \mathrm{m}$ nylon mesh to remove debris. The filtered cell suspension was incubated with $\alpha$-MEM supplemented with $10 \%$ FBS for $24 \mathrm{~h}$. After incubation, nonadherent cells were removed, and adherent cells, comprised of ATSCs, were cultured and expanded for further analysis. ATSCs that were passaged less than three were used for all experiments. The culture medium was changed every other day.

\section{Analysis of ATSC proliferation}

ATSCs were seeded into 96-well plates at a density of 700 cells/well. Cells were treated with the indicated concentrations of FGF-2 (R\&D Systems, Minneapolis, MN), betamethasone (BET), DEX, hydrocortisone (HYD), prednisolone (PRE) (Sigma, St. Louis, MO), or a combination of FGF-2 and steroid for 3,5 , or 7 days. Proliferation was measured using a proliferation assay kit (Promega, Madison, WI), according to manufacturer's instructions. Optical density at $490 \mathrm{~nm}$ was measured with an ELISA plate reader (BioRad, Hercules, CA). Cell number was obtained using a hemocytometer.

\section{Flow cytometric analysis}

ATSCs were incubated with FGF-2 and DEX in $\alpha$-MEM supplemented with $10 \%$ FBS for 5 days. Cells were harvested with $0.25 \%$ trypsin/EDTA and incubated in $1 \%$ bovine serum albumin (BSA) containing $100 \mu \mathrm{L}$ of a solution of monoclonal antibody directed against CD29, CD44, CD90, CD105, CD146, or CD34 (BD Pharmingen, Palo Alto, CA) for $30 \mathrm{~min}$ at $4^{\circ} \mathrm{C}$. The cells were centrifuged at $1200 \mathrm{~g}$ for $5 \mathrm{~min}$ and then washed with $1 \%$ BSA. The resuspended cells were fixed for $30 \mathrm{~min}$ in ice-cold $4 \%$ formaldehyde. After fixation, the cells were washed with 1\% BSA, and then analyzed using a FACSAria Sorting Flow Cytometer (Beckton Dickinson, Franklin Lakes, NJ).

\section{Multilineage differentiation of ATSCs}

ATSCs that were expanded in the presence of FGF-2 and DEX for 5 days were replated into 48-well plates at a density of $1 \times 10^{4}$ cells/well. On the following day, osteoblastic differentiation was induced by treatment with osteogenic medium ( $\alpha$-MEM supplemented with $10 \%$ FBS, $50 \mu \mathrm{g} / \mathrm{mL}$ $\alpha$-ascorbic acid, $10 \mathrm{mM} \beta$-glycerophosphate, and $10 \mathrm{nM}$ DEX) for 14 days. Osteoblastic differentiation was assessed using alizarin red S (AR-S) staining. AR-S deposits were recovered by incubating the cells in a solution of $10 \%$ cetylpyridinium chloride (Sigma) in $10 \mathrm{mM}$ sodium phosphate ( $\mathrm{pH}$ 7.0) for $15 \mathrm{~min}$ at room temperature. The amount of AR-S was

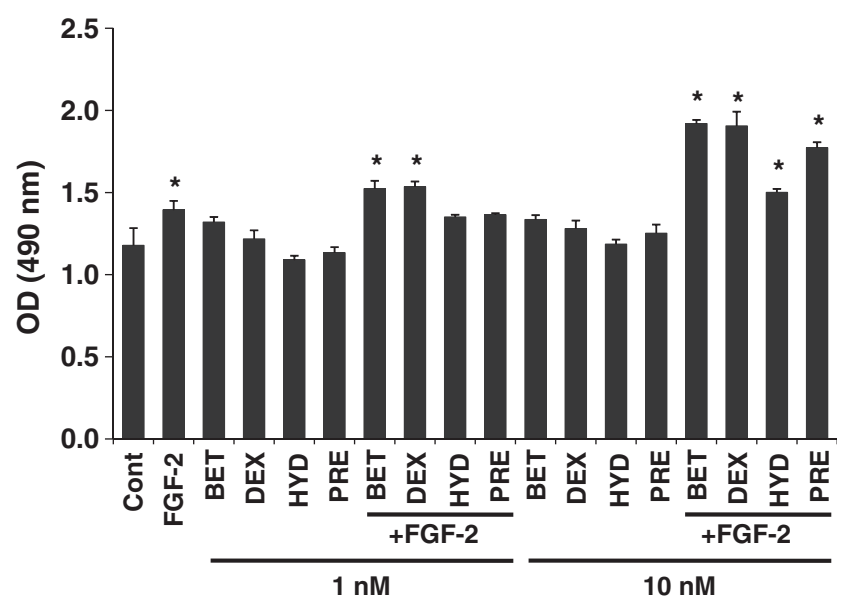

FIG. 1. Induction of proliferation of adipose tissue-derived mesenchymal stromal cells (ATSCs) by fibroblast growth factor-2 (FGF-2) and steroids. ATSCs were cultured in the presence of FGF-2 $(1 \mathrm{ng} / \mathrm{mL})$ and the indicated concentrations of steroid (BET, DEX, HYD, and PRE) for 7 days. Proliferation assays were performed as described in Materials and Methods. ${ }^{*} p<0.05$ as compared to untreated control cells. BET, betamethasone; DEX, dexamethasone; HYD, hydrocortisone; PRE, prednisolone; OD, optical density. 
A
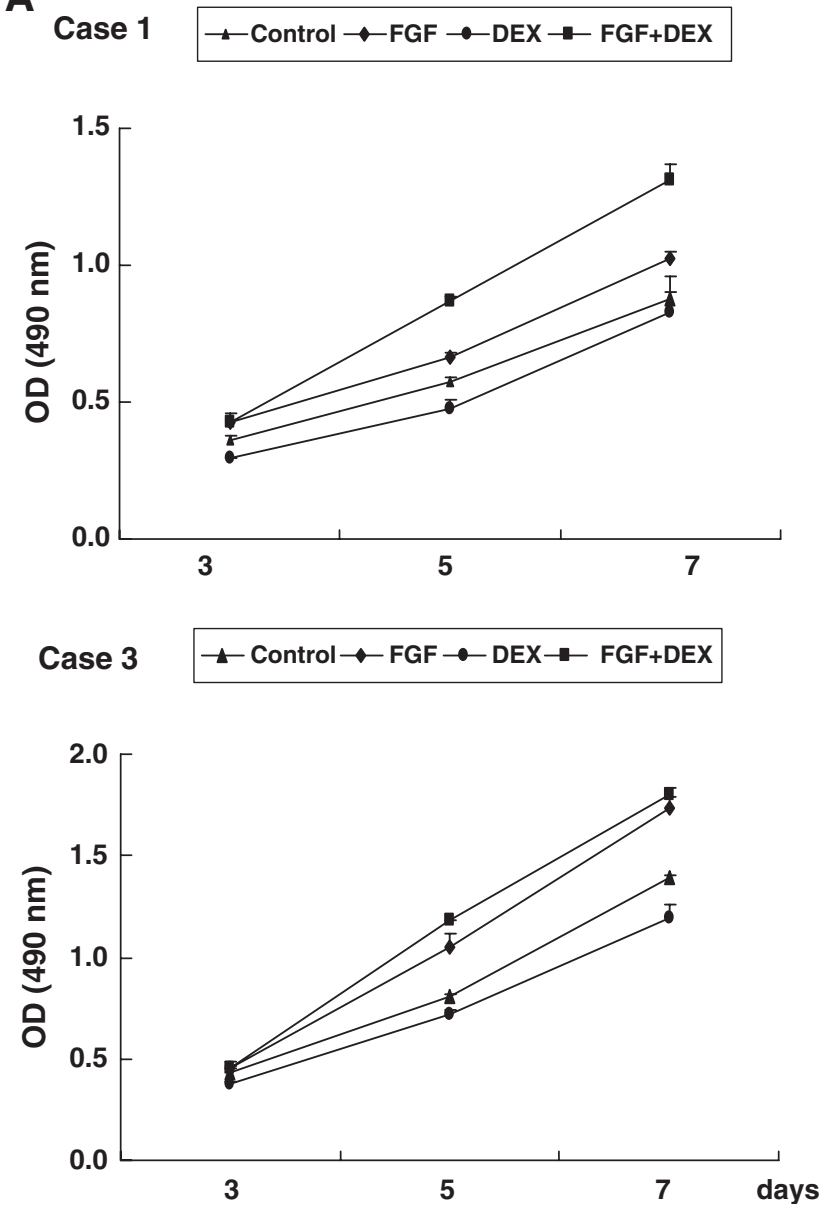
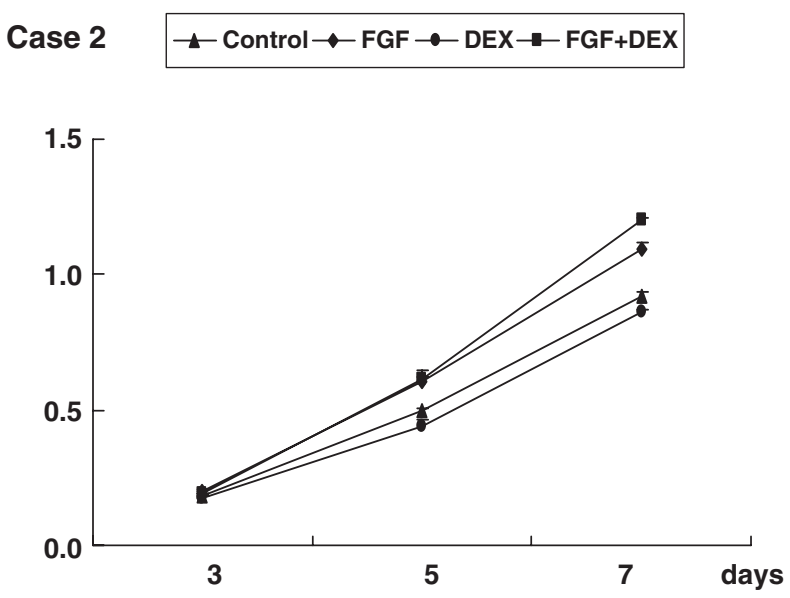

B

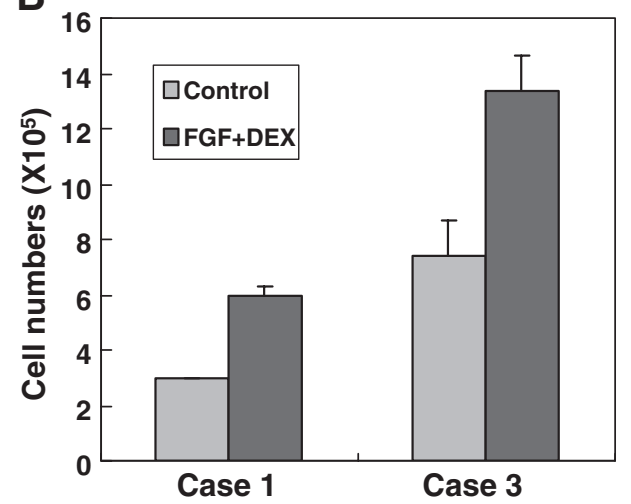

FIG. 2. Induction of proliferation of ATSCs derived from three independent donors by FGF-2 and DEX. ATSCs were cultured with FGF-2 (1 ng/mL), DEX (10 nM), or FGF-2 plus DEX for 3, 5, and 7 days. (A) Proliferation assays were carried out as described in Materials and Methods. Data represent the means \pm standard error (SE) of three independent experiments $(n=3)$. Proliferation of ATSCs by FGF-2 $(1 \mathrm{ng} / \mathrm{mL})+$ DEX $(10 \mathrm{nM})$ was significantly increased at 5 and 7 days of culture compared to control $\left({ }^{*}, p<0.05\right)$. (B) ATSCs were cultured with or without FGF-2 $(1 \mathrm{ng} / \mathrm{mL})$ plus DEX (10 nM) for 5 days, and cell number was measured using a hemocytometer.

determined by measuring absorbance at $570 \mathrm{~nm}$. Adipogenic differentiation was induced by treatment with adipogenic medium ( $\alpha$-MEM supplemented with 10\% FBS, $1 \mu \mathrm{M}$ DEX, $111 \mu \mathrm{g} / \mathrm{mL}$ isobutylmethylxanthine, $0.2 \mathrm{mM}$ indomethacin, and $10 \mu \mathrm{g} / \mathrm{mL}$ insulin) for 14 days. Adipogenesis was assessed using Oil red $\mathrm{O}$ staining. To obtain quantitative data, $300 \mu \mathrm{L}$ of isopropyl alcohol was added to the stained cells, and the amount of extracted dye was determined by absorbance at $540 \mathrm{~nm}$ using an ELISA plate reader.

\section{Western blot analysis}

Total cell lysate was prepared in RIPA buffer ( $50 \mathrm{mM}$ Tris, pH 7.4, 1\% NP-40, 150 mM NaCl, 1 mM EDTA, 1 mM PMSF, $1 \mathrm{mM} \mathrm{Na} 3 \mathrm{VO}_{4}, 1 \mathrm{mM} \mathrm{NaF}$, pepstatin $1 \mathrm{~g} / \mathrm{mL}$, and aprotinin $1 \mathrm{~g} / \mathrm{mL})$, and equal amounts of protein $(20 \mu \mathrm{g})$ were separated by $8 \%$ SDS-PAGE. Proteins were transferred to a nitrocellulose membrane. The membrane was incubated in a solution of $5 \%$ skim milk in Tris buffered saline containing Tween-20 (20 mM Tris, $\mathrm{pH} 7.6,137 \mathrm{mM} \mathrm{NaCl}$, and $0.1 \%$ Tween-20) and then incubated with primary antibodies directed against phosphorylated (phospho)-p38 (BD Pharmingen), phospho-Src, Src (Cell signaling Technology, Danvers,
MA), extracellular signal-regulated kinase (ERK), c-Jun $\mathrm{N}$ terminal kinase (JNK), or p38 (Santa Cruz, Santa Cruz, CA), as indicated. The membranes were incubated with peroxidase-conjugated secondary anti-mouse or anti-rabbit immunoglobulin (IgG) (Zymed, South San Francisco, CA), and immunoreactive proteins were detected using chemiluminescence and X-ray film.

TABle 1. Expression of MSC Markers on the Surface of ATSCs Expanded in the Presence of FGF-2, DEX, OR FGF-2 Plus DEX

\begin{tabular}{lcccc}
\hline Marker & Control & FGF-2 & DEX & FGF-2 + DEX \\
\hline CD29 & 97.6 & 98.2 & 98.3 & 98.3 \\
CD44 & 98.3 & 98.6 & 98.0 & 98.3 \\
CD90 & 98.0 & 97.8 & 97.8 & 97.0 \\
CD105 & 99.6 & 99.7 & 99.4 & 99.7 \\
CD146 & 9.3 & 8.9 & 7.3 & 4.6 \\
CD34 & 0.7 & 0.4 & 0.6 & 0.4 \\
\hline
\end{tabular}

MSCs, mesenchymal stem cells; ATSCs, adipose tissue-derived mesenchymal stromal cells; FGF-2, fibroblast growth factor-2; DEX, dexamethasone. 


\section{Statistical analysis}

Statistical analysis was performed using SPSS 11.0. Data were analyzed using one-way ANOVA, and a Duncan's Multiple Range Test was also adapted as a post hoc test. A pvalue of less than 0.05 was considered statistically significant.

\section{Results}

\section{Effect of steroids and FGF-2 on the proliferation of ATSCs}

To investigate the effect of steroids alone or in combination with a low concentration of FGF-2 $(1 \mathrm{ng} / \mathrm{mL})$ on the proliferation of ATSCs, cells were treated with varying amounts of steroid, with or without FGF-2, for 7 days. Treatment of ATSCs with 1 or $10 \mathrm{nM}$ BET, DEX, HYD, or PRE had no obvious stimulatory effect on proliferation.
However, when combined with FGF-2, 1 nM BET or DEX enhanced optical density (OD) by $29.3 \%$ and $30.3 \%$, respectively $(p<0.05)$, as compared to control (Fig. 1), indicating stimulation of the proliferation of ATSCs by these combinations. The stimulatory effect of steroids plus FGF-2 was greater in the presence of $10 \mathrm{nM}$ BET and DEX (increases of OD by $62.9 \%$ and $61.7 \%$, respectively, $p<0.05$ ) (Fig. 1 ). The effect of FGF-2 plus other steroids (HYD and PRE) on the proliferation of ATSCs was not prominent. These results suggested that FGF-2 and DEX or BET act in a cooperative manner to stimulate the proliferation of ATSCs.

\section{Effect of FGF-2 and DEX on the proliferation of donor-derived ATSCs}

To confirm the stimulatory effect of FGF-2 and steroids on the proliferation of ATSCs, ATSCs were isolated from

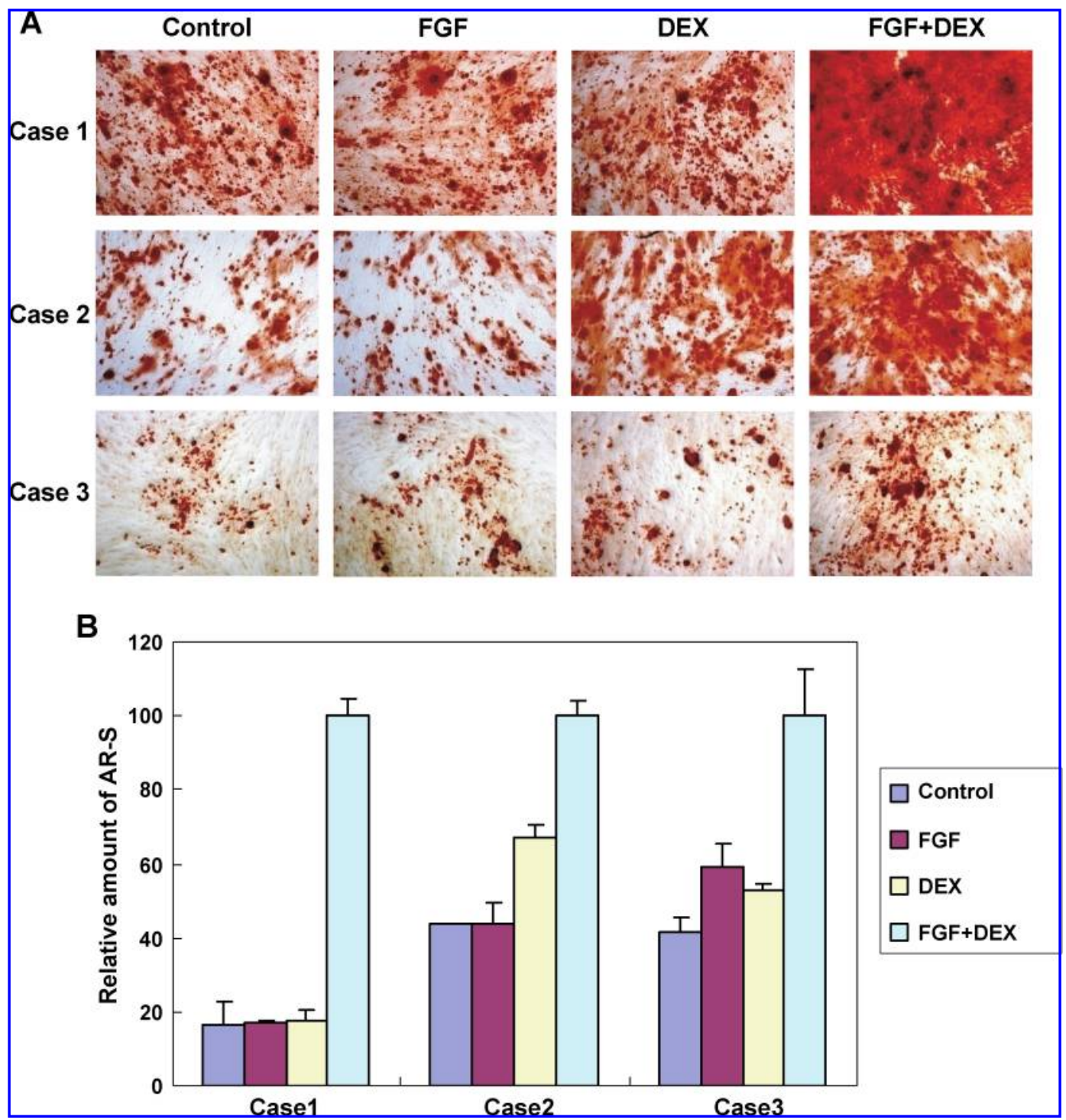

FIG. 3. Osteoblastic differentiation of ATSCs expanded in the presence of FGF-2 and DEX. ATSCs were cultured with FGF-2 $(1 \mathrm{ng} / \mathrm{mL}), \mathrm{DEX}(10 \mathrm{nM})$, or FGF-2 plus DEX for 5 days. The expanded cells were reseeded and treated with osteogenic medium for an additional 14 days to induce osteoblastic differentiation. (A) Calcium deposition was visualized by staining with alizarin red S (AR-S) solution (original magnification, 40×). (B) AR-S was extracted using 10\% (w/v) cetylpyridinium chloride and quantified by absorbance at $570 \mathrm{~nm}(\mathrm{OD} 570 \mathrm{~nm}$ ). Color images available online at www.liebertonline.com/ten. 
three independent donors and treated with FGF-2 $(1 \mathrm{ng} / \mathrm{mL})$, DEX (10 nM), or FGF-2 plus DEX for 3, 5, or 7 days. Treatment with FGF-2 alone increased the proliferation of ATSCs by an average of $22 \%$ and $20 \%$ at days 5 and 7 , respectively, as compared to control, whereas proliferation was increased by an average of $40 \%$ and $36.5 \%$ at days 5 and 7 , respectively, by FGF-2 plus DEX, as compared to untreated cells (Fig. 2A). Treatment with FGF-2 plus DEX increased the proliferation of ATSCs by an average of $14.5 \%$ and $13.6 \%$ at days 5 and 7, respectively, as compared to FGF-2 treatment alone (Fig. 2A). To confirm these results, we carried out an analysis of cell number. After treatment with FGF-2 and DEX, the number of ATSCs increased by $81-103 \%$ as compared to control (Fig. 2B). These results demonstrated that combined treatment with low concentrations of FGF-2 and DEX strongly enhances the proliferation of ATSCs in vitro.

\section{Expression levels of MSC markers in ATSCs cultured with FGF-2 and DEX}

To determine whether ATSCs that were expanded in the presence of FGF-2 and DEX maintained the expression of MSC cell surface markers, ATSCs were cultured with FGF-2 and DEX for 5 days, and the expression of CD29, CD44, CD90, CD105, and CD146 was examined by flow cytometry. More than $97 \%$ of cells that were expanded in the presence of FGF-2 and DEX were positive for CD29, CD44, CD90, and CD105, which was similar to untreated ATSCs (Table 1). However, CD146-positive cells were only between $4.5 \%$ and $9.3 \%$ (Table 1). This result is consistent with a previous study, demonstrating that CD146 expression was decreased during passage. ${ }^{22}$ Therefore, ATSCs that were expanded in vitro in the presence of FGF-2 and DEX expressed MSC markers, including CD29, CD44, CD90, CD105, and CD146.

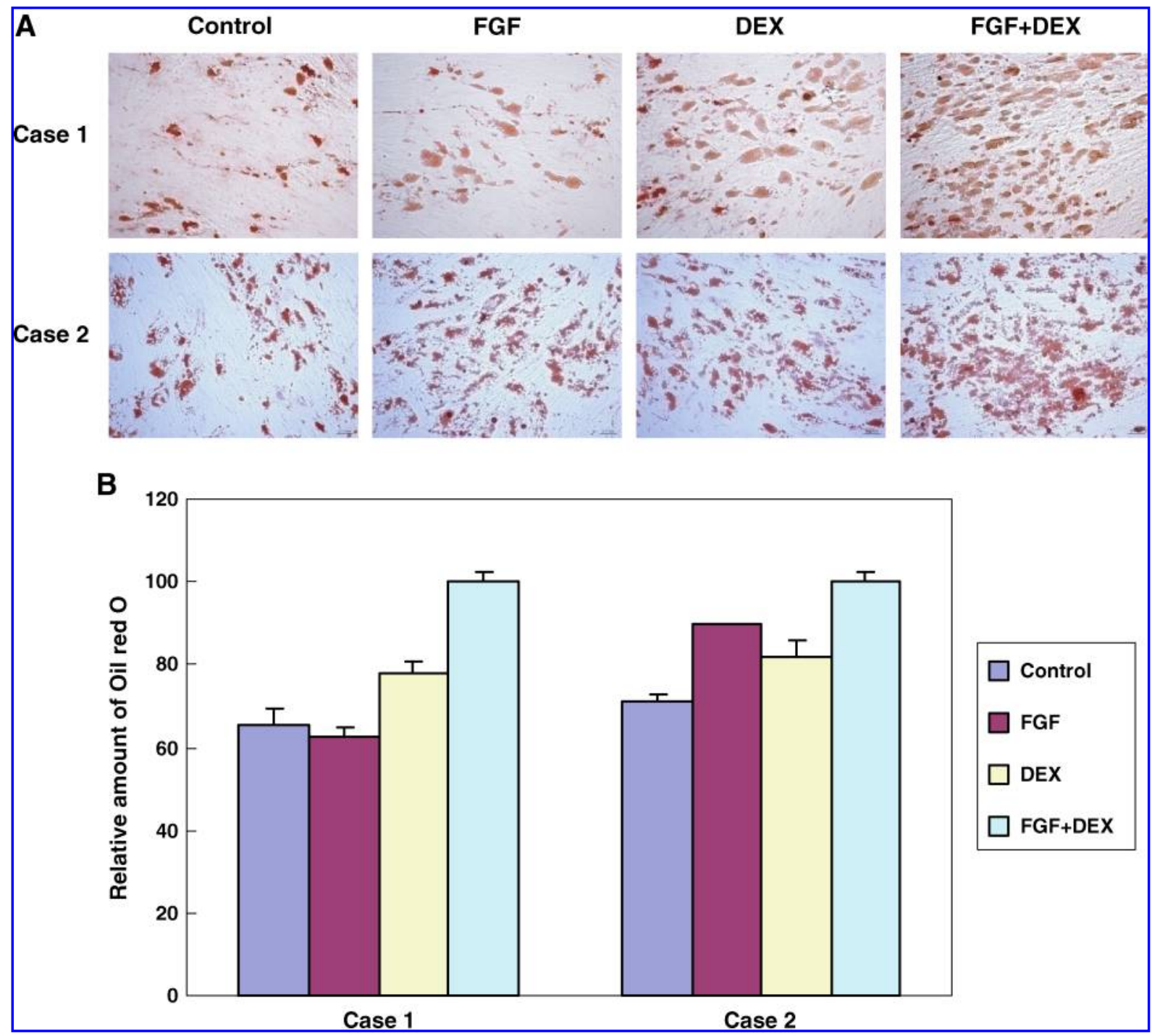

FIG. 4. Adipogenic differentiation of ATSCs expanded in the presence of FGF-2 and DEX. ATSCs were cultured with FGF-2 $(1 \mathrm{ng} / \mathrm{mL})$, DEX $(10 \mathrm{nM})$, or FGF-2 plus DEX for 5 days. The expanded cells were reseeded and treated with adipogenic medium for an additional 14 days. (A) Oil droplets were visualized by staining with oil red $\mathrm{O}$ solution (original magnification, $40 \times$ ). (B) Stain was extracted using isopropyl alcohol, and the amount of oil red $\mathrm{O}$ was quantified by OD $570 \mathrm{~nm}$. Color images available online at www.liebertonline.com/ten. 
Effect of FGF-2 and DEX on osteoblastic and adipogenic differentiation of ATSCS

To determine whether ATSCs cultured in vitro with FGF-2 and DEX maintained their multi-lineage differentiation potential, ATSCs were cultured in the absence (control cells) or presence of FGF-2 and DEX for 5 days. Expanded cells were collected, reseeded, and then cultured for an additional 14 days in osteogenic or adipogenic medium. ATSCs that were first expanded with FGF-2 and DEX were capable of differentiating into either osteoblastic (Fig. 3) or adipogenic cells (Fig. 4). Of note, ATSCs expanded in the presence of FGF-2 and DEX exhibited increased levels of osteoblastic and adipogenic differentiation as compared to control cells (Figs. 3 and 4). These results indicated that the expansion of ATSCs in the presence of FGF-2 and DEX maintains their osteogenic and adipogenic differentiation potential.

\section{Tyrosine phosphorylation of Src kinase} in ATSCs exposed to FGF-2 and DEX

To begin to elucidate the signaling pathways that were involved in the stimulatory effect of FGF-2 and DEX on the proliferation of ATSCs, cells were treated with FGF-2 and DEX for 1, 3, or 5 days, and the level of phosphorylation of key signaling molecules was examined by Western blot. Tyrosine phosphorylation of Src kinase was significantly increased by treatment with FGF-2 and DEX, whereas that of
JNK, p38, and ERK was decreased (Fig. 5). These results suggested the potential involvement of Src kinase in the stimulation of proliferation and subsequent osteoblastic differentiation of ATSCs by FGF-2 and DEX.

\section{Effect of Src kinase inhibitors on FGF-2 and DEX-induced proliferation of ATSCs}

To further examine the involvement of Src or Src family kinases in the stimulation of proliferation of ATSCs, cells were cultured in the presence of FGF-2 and DEX, with or without (control cells) selective Src family kinase inhibitors (SU6656 and src kinase inhibitor I). The proliferation of ATSCs in the presence of SU6656 (Fig. 6A) and src kinase inhibitor I (Fig. 6B) was significantly decreased in a dosedependent manner as compared to control cells. These results were consistent with the increased tyrosine phosphorylation of Src by FGF-2 and DEX, and strongly suggested that the stimulation of proliferation of ATSCs by FGF-2 and DEX involves Src kinase.

\section{Effect of Src kinase inhibitors on FGF-2 and $D E X$-induced osteoblastic and adipogenic differentiation of ATSCS}

To determine whether the inhibition of Src kinase affected osteoblastic and adipogenic differentiation, ATSCs were cultured with FGF-2 and DEX in the presence or absence

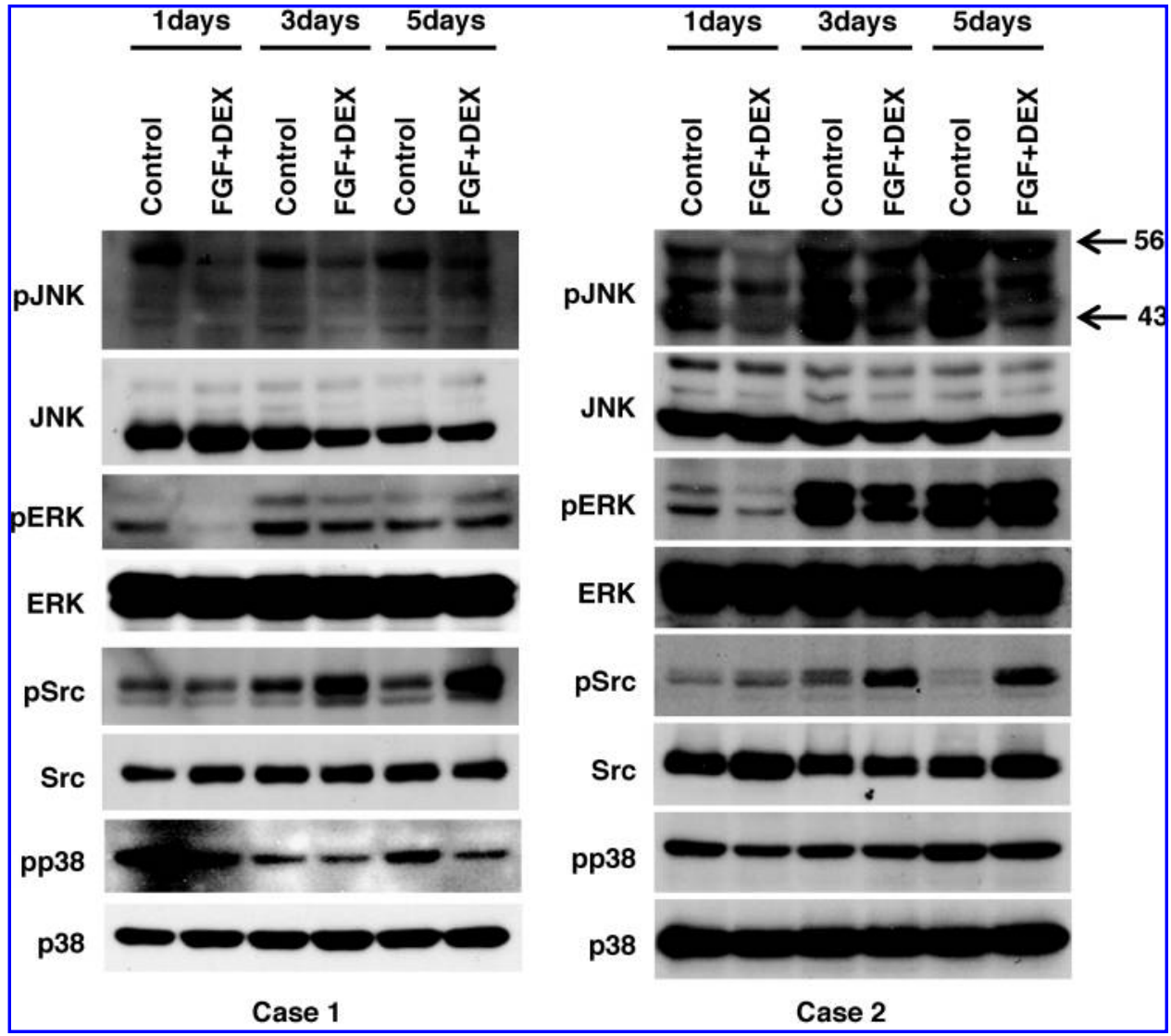

FIG. 5. Protein phosphorylation in ATSCs expanded in the presence of FGF-2 and DEX. ATSCs were treated with FGF-2 plus DEX for 1, 3, or 5 days, and the levels of phosphorylation of JNK, ERK, Src, and p38 were analyzed by Western blot. The phosphorylation of Src kinase was increased by FGF-2 and DEX. 
A Case 1

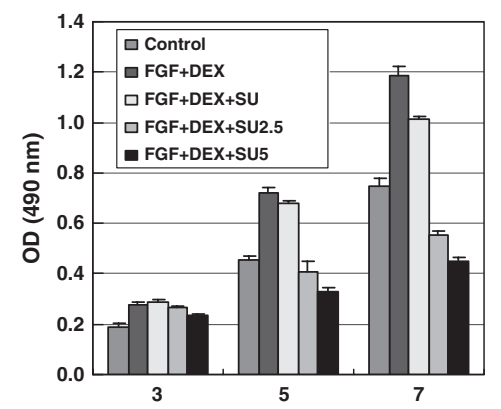

B Case 1

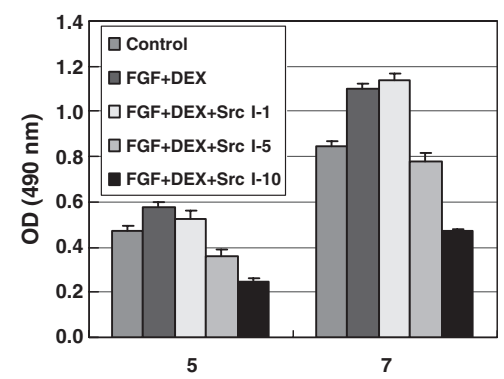

Case 2

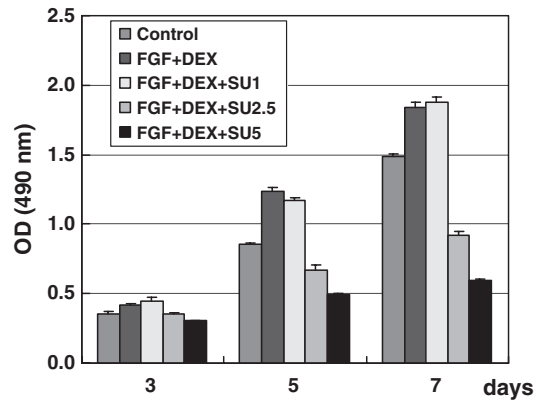

Case 2

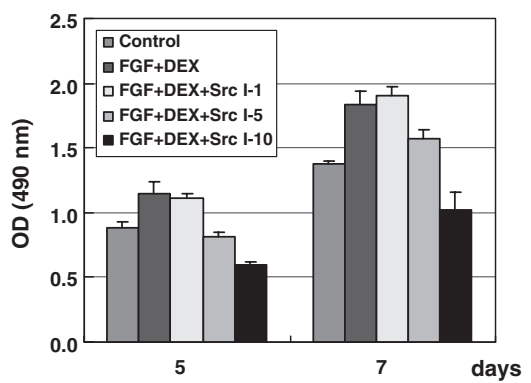

FIG. 6. Effect of Src family kinase inhibitors (SU6656 and src kinase inhibitor I) on FGF-2 and DEX-induced proliferation of ATSCs. ATSCs were cultured with FGF-2 $(1 \mathrm{ng} / \mathrm{mL})$ plus DEX $(10 \mathrm{nM})$ in the presence or absence of the indicated concentrations of SU6656 (A) or src kinase inhibitor I (B) for 3, 5, or 7 days. Proliferation was measured as described in Materials and Methods. Data represents the means \pm SE $(n=3)$.

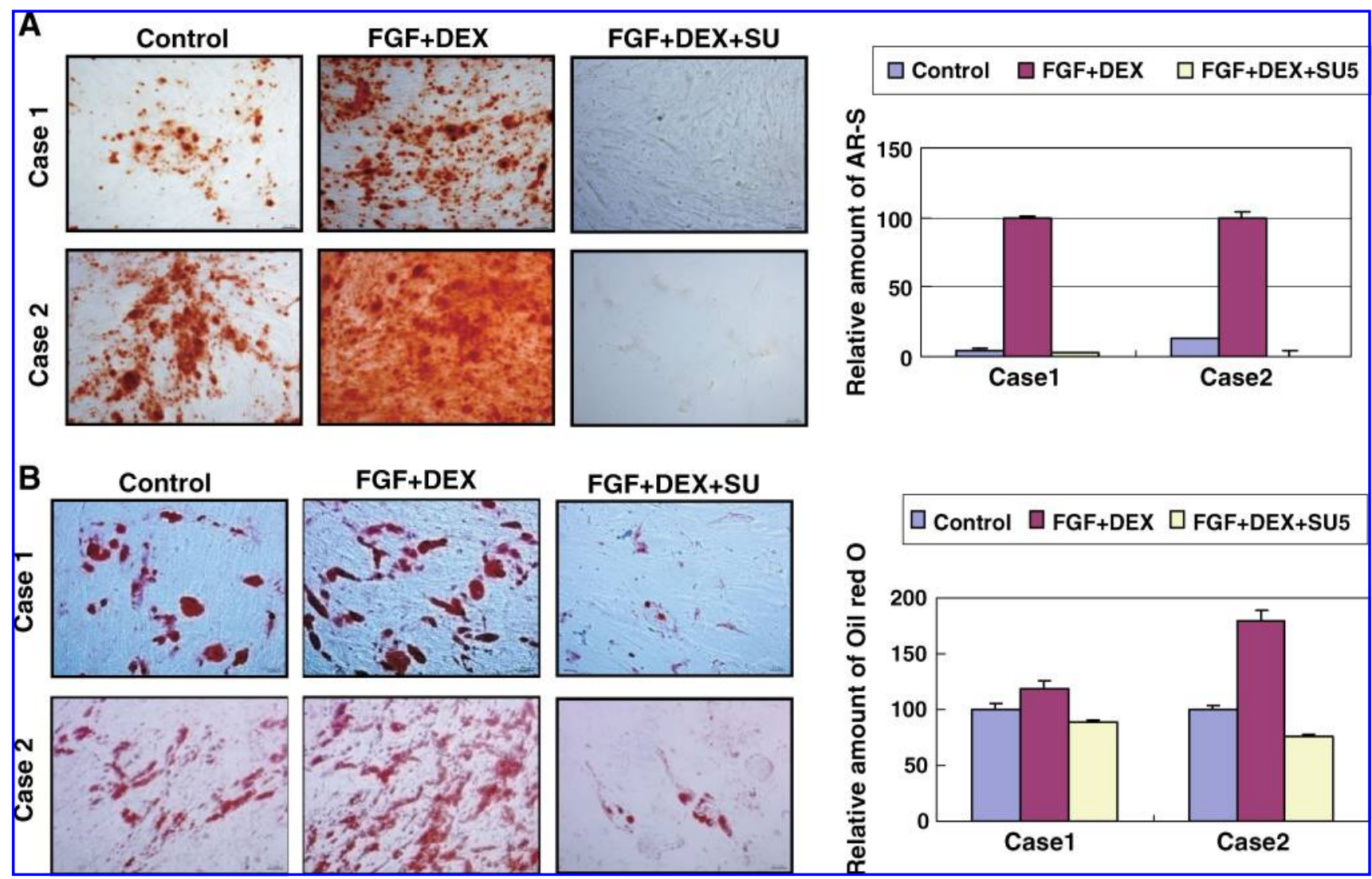

FIG. 7. Effect of SU6656 on FGF-2 and DEX-induced osteoblastic and adipogenic differentiation of ATSCs. ATSCs were cultured with FGF-2 $(1 \mathrm{ng} / \mathrm{mL})$ plus DEX $(10 \mathrm{nM})$ in the presence or absence of SU6656 $(5 \mu \mathrm{M})$ for 5 days. Expanded cells were reseeded and treated with either osteogenic or adipogenic medium. (A) Calcium deposition was assessed by staining with AR-S solution (original magnification, 40×). AR-S staining was quantified as described for Figure 3B (right panel). (B) Oil droplets were visualized by staining with oil red $\mathrm{O}$ solution (original magnification, 40×). (B) Oil red $\mathrm{O}$ staining was quantified as described for Figure 4B (right panel). Color images available online at www.liebertonline.com/ten. 
(control cells) of SU6656, and then the expanded ATSCs were cultured for an additional 14 days in osteogenic or adipogenic medium. The osteoblastic differentiation of ATSCs that were pretreated with SU6656 was decreased as compared to control cells, based on AR-S staining. Similar results were observed in two independent donor-derived ATSCs (Fig. 7A). The level of adipogenic differentiation was also decreased by SU6656, as assessed by oil red O staining (Fig. 7B). These results suggested that $\mathrm{Src}$ kinase modulates the osteoblastic and adipogenic differentiation potential of ATSCs.

\section{Discussion}

ATSCs have proven to be a useful source of cells for the treatment of defective tissues by tissue engineering. However, to be useful for clinical applications, particularly for the treatment of large lesions, it will be necessary to first expand ATSCs in vitro. Previously, we and others have shown that FGF-2 efficiently enhances the proliferation of BMSCs. ${ }^{9-11,23}$ A recent paper by Zaragosi et al. showed that FGF-2 plays a critical role in the self-renewal of ATSCs. ${ }^{14}$ A low concentration of FGF-2 may not induce chromosomal abnormality in MSCs. ${ }^{24}$ Therefore, FGF-2 appears to be an essential growth factor in the regulation of MSC proliferation and selfrenewal. Here, we investigated ways to enhance the growth of ATSCs using a combination of FGF and steroids, and found that FGF-2 in combination with BET or DEX substantially enhances the growth of ATSCs in vitro (Fig. 1). The enhanced growth of ATSCs by FGF-2 and DEX was confirmed in ATSCs from three independent donors (Fig. 2). Previous reports by us and others using BMSCs and cementoblasts have shown that a combination of FGF-2 and DEX modulates the proliferation and biomineralization of BMSCs. However, the underlying molecular mechanism of this enhanced proliferation remains largely unknown. ${ }^{14,25,26}$

FGF-2 in combination with DEX enhanced the proliferation of ATSCs derived from three independent donors (Fig. 2). Because FGF-2-induced proliferation was enhanced by DEX, it is reasonable to hypothesize that FGF-2-induced signaling pathways are potentiated by DEX, thus resulting in enhanced ATSC proliferation. When we examined whether DEX enhanced the expression of FGF receptors (FGFRs) as a mechanism of amplification of FGF signaling, we found that the expression levels of FGFRI, II, and IV were unaffected by FGF-2 and DEX (Supplemental Fig. S1A, available online at www.liebertonline.com/ten). Although FGFRIII was induced by DEX, its expression was decreased by FGF$2+$ DEX, suggesting that modulation of FGFRIII expression may not contribute to the enhanced proliferation of ATSCs. We also examined the phosphorylation levels of key signaling molecules involved in cellular proliferation, such as JNK, p38, ERK, and Src kinase. As shown in Figure 5, the levels of phosphorylation of p38, ERK, and JNK were decreased by treatment with FGF-2 and DEX, while the phosphorylation of Src kinase was substantially increased. These results imply that Src kinase plays a role in the stimulatory effect of FGF-2 and DEX on the proliferation of ATSCs. Subsequent analysis of cell proliferation in the presence of inhibitors of Src kinase confirmed that Src is involved in FGF-2 and DEX-induced stimulation of ATSC proliferation (Figs. 6 and 7).

ATSCs that were expanded in the presence of FGF-2 and DEX were able to differentiate into either osteoblasts or adi- pocytes. Although we did not analyze the differentiation of the expanded ATSCs along other lineages, these results suggest that FGF-2 and DEX maintain or preserve the multilineage potential of ATSCs that are expanded in vitro. Significantly, ATSCs that were expanded in vitro with FGF-2 and DEX exhibited a higher tendency to differentiate into both osteoblasts and adipocytes. These results were in agreement with previous reports in which a combination of FGF-2 and DEX promoted the osteogenic commitment of BMSCs. ${ }^{18,19,27}$ In the current study, we also detected the expression of ALP in cells that were treated with FGF-2 and DEX, which indicates the commitment of ATSCs to an osteogenic lineage (Supplemental Fig. S1B). Treatment with FGF-2 plus DEX also promoted the commitment of ATSCs to the adipogenic lineage. The mRNA levels of PPAR $\gamma 2$, which plays an important role in adipocyte differentiation, were increased in cells cultured with FGF-2 and DEX for 5 days (Supplemental Fig. S1B). Thus, FGF-2 and DEX increased the osteogenic potential, as well as the adipogenic potential of ATSCs.

The inhibition of Src kinase resulted in the suppression of osteoblastic and adipogenic differentiation in ATSCs (Figs. 6 and 7). The tyrosine phosphorylation of Src kinase increased during FGF-2 and DEX-induced proliferation of ATSCs (Fig. 5), and treatment with inhibitors of Src family kinases suppressed the proliferation of ATSCs (Fig. 6). These results suggest that the osteogenic and adipogenic differentiation of ATSCs is modulated by the proliferative capacity of the cells. In fact, when the number of cells that were incubated in osteogenic medium was increased, we observed an enhancement of differentiation of ATSCs along the osteoblastic lineage (Supplemental Fig. S2, available online at www.liebertonline.com/ ten). These results suggest that the inhibition of Src kinase causes the suppression of cell growth and subsequent differentiation of ATSCs along the osteoblastic or adipocytic lineage.

In summary, we have demonstrated that treatment of ATSCs with a combination of FGF-2 and DEX is an efficient method for expanding these cells in vitro and, importantly, can preserve the osteogenic and adipogenic potential of the cells. We also demonstrated that Src kinase is involved in the stimulation of proliferation of ATSCs.

\section{Acknowledgments}

This work was supported by a grant from the Ministry of Health and Welfare [0405-BO01-0204-0006] and by a Korea Science and Engineering Foundation (KOSEF) grant funded by the Korea government (MOST); Grant Numbers: M10646020001-06N4602-00110 and No. R01-2006-000-10756-0).

\section{Disclosure Statement}

No competing financial interests exist.

\section{References}

1. Gimble, J., and Guilak, F. Adipose-derived adult stem cells: isolation, characterization, and differentiation potential. $\underline{\text { Cyto- }}$ therapy 5, 362, 2003.

2. Halvorsen, Y.D., Bond, A., Sen, A., Franklin, D.M., LeaCurrie, Y.R., Sujkowski, D., Ellis, P.N., Wilkison, W.O., and Gimble, J.M. Thiazolidinediones and glucocorticoids synergistically induce differentiation of human adipose tissue stromal cells: biochemical, cellular, and molecular analysis. Metabolism 50, 407, 2001. 
3. Zuk, P.A., Zhu, M., Ashjian, P., de Ugarte, D.A., Huang, J.I., Mizuno, H., Alfonso, Z.C., Fraser, J.K., Benhaim, P., and Hedrick, M.H. Human adipose tissue is a source of multipotent stem cells. Mol Biol Cell 13, 4279, 2002.

4. Zuk, P.A., Zhu, M., Mizuno, H., Huang, J., Futrell, J.W., Katz, A.J., Benhaim, P., Lorenz, H.P., and Hedrick, M.H. Multilineage cells from human adipose tissue: implications for cell-based therapies. Tissue Eng 7, 211, 2001.

5. Gronthos, S., Franklin, D.M., Leddy, H.A., Robey, P.G., Storms, R.W., and Gimble, J.M. Surface protein characterization of human adipose tissue-derived stromal cells. I Cell Physiol 189, 54, 2001.

6. Hattori, H., Masuoka, K., Sato, M., Ishihara, M., Asazuma, T., Takase, B., Kikuchi, M., Nemoto, K., and Ishihara, M. Bone formation using human adipose tissue-derived stromal cells and a biodegradable scaffold. J Biomed Mater Res B Appl Biomater 76, 230, 2006.

7. Peterson, B., Zhang, J., Iglesias, R., Kabo, M., Hedrick, M., Benhaim, P., and Lieberman, J.R. Healing of critically sized femoral defects, using genetically modified mesenchymal stem cells from human adipose tissue. Tissue Eng 11, 120, 2005.

8. Ornitz, D.M., and Marie, P.J. FGF signaling pathways in endochondral and intramembranous bone development and human genetic disease. Genes Dev 16, 1446, 2002.

9. Martin, I., Muraglia, A., Campanile, G., Cancedda, R., and Quarto, R. Fibroblast growth factor-2 supports ex vivo expansion and maintenance of osteogenic precursors from human bone marrow. Endocrinology 138, 4456, 1997.

10. Oliver, L.J., Rifkin, D.B., Gabrilove, J., Hannocks, M.J., and Wilson, E.L. Long-term culture of human bone marrow stromal cells in the presence of basic fibroblast growth factor. Growth Factors 3, 231, 1990.

11. Pri-Chen, S., Pitaru, S., Lokiec, F., and Savion, N. Basic fibroblast growth factor enhances the growth and expression of the osteogenic phenotype of dexamethasone-treated human bone marrow-derived bone-like cells in culture. Bone 23, 111, 1998.

12. Chiou, M., Xu, Y., and Longaker, M.T. Mitogenic and chondrogenic effects of fibroblast growth factor-2 in adipose-derived mesenchymal cells. Biochem Biophys Res Commun 343, 644, 2006.

13. Quarto, N., and Longaker, M.T. FGF-2 inhibits osteogenesis in mouse adipose tissue-derived stromal cells and sustains their proliferative and osteogenic potential state. Tissue Eng 12, 1405, 2006.

14. Zaragosi, L.E., Ailhaud, G., and Dani, C. Autocrine FGF2 signaling is critical for self-renewal of human multipotent adipose-derived stem cells. Stem Cells 24, 2412, 2006.

15. Bellows, C.G., Heersche, J.N., and Aubin, J.E. Determination of the capacity for proliferation and differentiation of osteoprogenitor cells in the presence and absence of dexamethasone. Dev Biol 140, 132, 1990.

16. Cheng, S.L., Yang, J.W., Rifas, L., Zhang, S.F., and Avioli, L.V. Differentiation of human bone marrow osteogenic stromal cells in vitro: induction of the osteoblast phenotype by dexamethasone. Endocrinology 134, 277, 1994.

17. Pittenger, M.F., Mackay, A.M., Beck, S.C., Jaiswal, R.K., Douglas, R., Mosca, J.D., Moorman, M.A., Simonetti, D.W., Craig, S., and Marshak, D.R. Multilineage potential of adult human mesenchymal stem cells. Science 284, 143, 1999.

18. Frank, O., Heim, M., Jakob, M., Barbero, A., Schafer, D., Bendik, I., Dick, W., Heberer, M., and Martin, I. Real-time quantitative RT-PCR analysis of human bone marrow stromal cells during osteogenic differentiation in vitro. J Cell Biochem 85, 737, 2002.
19. Locklin, R.M., Oreffo, R.O., and Triffitt, J.T. Effects of TGFbeta and bFGF on the differentiation of human bone marrow stromal fibroblasts. Cell Biol Int 23, 185, 1999.

20. Muraglia, A., Martin, I., Cancedda, R., and Quarto, R. A nude mouse model for human bone formation in unloaded conditions. Bone 22, 131S, 1998.

21. Locklin, R.M., Williamson, M.C., Beresford, J.N., Triffitt, J.T., and Owen, M.E. In vitro effects of growth factors and dexamethasone on rat marrow stromal cells. Clin Orthop Relat Res 313, 27, 1995.

22. Mitchell, J.B., McIntosh, K., Zvonic, S., Garrett, S., Floyd, Z.E., Kloster, A., Di Halvorsen, Y., Storms, R.W., Goh, B., Kilroy, G., $\mathrm{Wu}, \mathrm{X}$., and Gimble, J.M. Immunophenotype of human adipose-derived cells: temporal changes in stromalassociated and stem cell-associated markers. Stem cells (Dayton, Ohio) 24, 376, 2006.

23. Shon, M., Lee, S., Kim, T., Kim, S., Khang, G., Son, Y., Shin, H., Kim, S., and Park, E. Effect of growth factors and dexamethasone on proliferation of bone marrow stromal cell. Tissue Eng Regen Med 3, 457, 2006.

24. Choumerianou, D.M., Dimitriou, H., Perdikogianni, C., Martimianaki, G., Riminucci, M., and Kalmanti, M. Study of oncogenic transformation in ex vivo expanded mesenchymal cells, from paediatric bone marrow. Cell Prolif 41, 909, 2008.

25. Hakki, S.S., Nohutcu, R.M., Hakki, E.E., Berry, J.E., Akkaya, M.S., and Somerman, M.J. Dexamethasone and basic-fibroblast growth factor regulate markers of mineralization in cementoblasts in vitro. J Periodontol 76, 1550, 2005.

26. Shon, M., Lee, S., Kim, T., You, C., Kim, S., Choung, P., Son, Y., Park, E., and Kim, S. Effect of FGF-2 and dexamethasone on proliferation and osteoblastic differentiation of bone marrow stromal cells growing under heat-inactivated autologous serum. Tissue Eng Regen Med 2, 356, 2005.

27. Walsh, S., Jefferiss, C., Stewart, K., Jordan, G.R., Screen, J., and Beresford, J.N. Expression of the developmental markers STRO-1 and alkaline phosphatase in cultures of human marrow stromal cells: regulation by fibroblast growth factor (FGF)-2 and relationship to the expression of FGF receptors 1-4. Bone 27, 185, 2000.

Address correspondence to: Eui Kyun Park, Ph.D.

Department of Pathology and Regenerative Medicine IHBR, School of Dentistry

Kyungpook National University 188-1 Samduk 2-ga, Jung-gu Daegu 700-412 Republic of Korea

E-mail: epark@knu.ac.kr

Shin-Yoon Kim, M.D., Ph.D. Department of Orthopaedic Surgery School of Medicine

Kyungpook National University 50 Samduk 2-ga, Jung-gu Daegu 700-712 Republic of Korea

E-mail: syukim@knu.ac.kr

Received: August 12, 2008

Accepted: January 22, 2009

Online Publication Date: March 9, 2009 



\section{This article has been cited by:}

1. Yongxing Liu, Diane R. Wagner. 2012. Effect of expansion media containing fibroblast growth factor-2 and dexamethasone on the chondrogenic potential of human adipose-derived stromal cells. Cell Biology International 36:7, 611-615. [CrossRef]

2. Karthik Ramasamy, Hazera Khatun, Lee Macpherson, Mathew P. Caley, Justin Sturge, Ghulam J. Mufti, Stephen A. Schey, Yolanda Calle. 2012. Fluorescence-based experimental model to evaluate the concomitant effect of drugs on the tumour microenvironment and cancer cells. British Journal of Haematology n/a-n/a. [CrossRef]

3. Biomaterials and Manufacturing Methods for Scaffold in Regenerative Medicine 3-47. [CrossRef]

4. Bodhraj Acharya, So-Young Chun, Shin-Yoon Kim, Cheil Moon, Hong-In Shin, Eui Kyun Park. 2012. Surface immobilization of MEPE peptide onto HA/\#-TCP ceramic particles enhances bone regeneration and remodeling. Journal of Biomedical Materials Research Part B: Applied Biomaterials n/a-n/a. [CrossRef]

5. Ling Sun, Shaobo Yu, Hongyang Wang, Bin Fan, Bang Liu. 2011. NUDT6, the FGF-2's antisense gene, showed associations with fat deposition related traits in pigs. Molecular Biology Reports . [CrossRef]

6. So Young Chun, Hyo Jung Lee, Young Ae Choi , Kyung Min Kim, Sang Heum Baek, Hyo Sang Park, Jae-Young Kim , Jung-Mo Ahn, Je-Yeol Cho, Dong-Woo Cho, Hong-In Shin, Eui Kyun Park . 2011. Analysis of the Soluble Human Tooth Proteome and Its Ability to Induce Dentin/Tooth Regeneration. Tissue Engineering Part A 17:1-2, 181-191. [Abstract] [Full Text HTML] [Full Text PDF] [Full Text PDF with Links] [Supplemental material]

7. Rashi Khanna-Jain, Annukka Vuorinen, George K.B. Sándor, Riitta Suuronen, Susanna Miettinen. 2010. Vitamin D3 metabolites induce osteogenic differentiation in human dental pulp and human dental follicle cells. The Journal of Steroid Biochemistry and Molecular Biology 122:4, 133-141. [CrossRef]

8. Florelle Gindraux, Laurent Obert, Laurent Laganier, Laurence Barnouin. 2010. Industrial approach in developing an advanced therapy product for bone repair. Journal of Tissue Engineering and Regenerative Medicine 4:3, 194-204. [CrossRef]

9. Ludovic Zimmerlin, Vera S. Donnenberg, Melanie E. Pfeifer, E. Michael Meyer, Bruno Péault, J. Peter Rubin, Albert D. Donnenberg. 2009. Stromal vascular progenitors in adult human adipose tissue. Cytometry Part A 9999A, NA-NA. [CrossRef] 